\title{
A propósito de "Dos sesiones del seminario Zubiri de 1978”
}

Estas sesiones son de singular interés para los estudiosos de la filosofía de $\mathbf{X}$. Zubiri, pero también para los neófitos, ya que pueden aportar algunas luces para leer en la perspectiva adecuada y enfatizar algunas cuestiones de la última obra de X. Zubiri. Asimismo, permiten apreciar el papel de interlocutor que juega 1. Ellacuría en la elaboración del planteamiento zubiriano y las cordiales discrepancias entre dos filósofos unidos por una profunda amistad y respeto mutuo. Diversos temas neurálgicos sobre los que creo que no se ha dicho todavía la última palabra son abordados aquí: ¿Cuál es el punto de partida de $\mathrm{X}$. Zubiri? ¿Es legítimo hablar de filosofía primera en X. Zubiri? Y de ser así, ¿qué deberíamos entender por ella? ¿Cuál es la diferencia estricta entre el realismo de $\mathrm{X}$. Zubiri y toda suerte de realismos metafísicos? ¿Cabe hablar de rompimiento entre la fenomenología y X. Zubiri? ¿En qué se diferencia y cuál es la relación que tiene en mente $X$. Zubiri entre la filosofía y los demás saberes? ¿Qué entiende X. Zubiri por inmediatez frente a todo idealismo? ¿Cuáles son definitivamente las etapas de la maduración de la filosofía zubiriana? ¿Llegó l. Ellacuría a instalarse en la radicalidad de la filosofía última de X. Zubiri? Estas cuestiones no agotan, ni mucho menos, toda la riqueza de estas dos sesiones, pero son problemas a menudo debatidos y que este texto quizás ayude a perfilar, debatir y precisar.

\section{La filosofia primera de X. Zubiri}

En las dos sesiones transcritas aparece con claridad que ni el escenario de la conciencia (Husserl) y las filosofías de la subjetividad, ni el del ser heideggeriano, ni la vida o ejecutividad orteguiana son para X. Zubiri el escenario radical. El escenario radical es el de la formalidad de realidad. Pero si esta formalidad de realidad sólo es accesible en la aprehensión, entonces parece que la filosofía primera más que en un análisis trascendental de la realidad consiste en una moología o estudio de los actos de aprehensión. Determinar en qué consiste la 
filosofía primera o el escenario radical de la filosofía de X. Zubiri parece, en principio, algo elemental porque, aunque en sus obras publicadas no habla en ningún sitio de filosofía primera, en aquellos lugares donde se refiere explícitamente a ella, por ejemplo en el curso de 1954 sobre filosofía primera, lo hace para referirse a la metafísica o análisis trascendental de la realidad. Sin embargo, la cuestión es más arriesgada de lo que parece a primera vista. La cosa se complica cuando, a partir de 1976, X. Zubiri empieza a explicitar su filosofía de la inteligencia. X. Zubiri advierte expresamente del equívoco mortal que supone pensar que las cosas reales son cosas reales fuera de la intelección y que no hacen sino actualizarse en ésta. Justamente en la primera de estas sesiones nos advierte: "Claro esto no lo diría yo jamás, es un equívoco mortal. ¿De dónde sé yo que hay otras cosas?"' . Este es un equívoco a menudo presente en la reflexión de algunos zubirianos anclados en Sobre la esencia, como si la filosofía de la inteligencia fuera sólo la vía de acceso a lo real, una propedéutica de la auténtica filosofía primera.

El problema es que $X$. Zubiri culmina su producción filosófica con una noología, esto es, con una investigación de lo que formalmente sea la inteligencia y justamente en esta obra, diversos estudiosos han apreciado una radicalización tal de determinadas nociones zubirianas, aun dentro de lo que X. Zubiri considera su última etapa (1945-1983), que los lleva a establecer a la noología como filosofía primera ${ }^{2}$. Juan Bañón ha subrayado, por ejemplo, que la noción de realidad de Sobre la esencia está muy cercana a la determinación de lo que es lo real independientemente de nuestra aprehensión. Al menos el grado de radicalidad no sería el mismo que en la Trilogía, donde no se inquiere ya qué sean las cosas más allá de su estamos presentes, sino que se intenta describir simplemente lo que nos está dado ${ }^{3}$ ¿ ¿No hay implícito en el pensamiento último de X. Zubiri una filosofía primera más radical que no es noológica ni metafísica? El impulso zubiriano abierto al desfallecimiento y al fracaso es probablemente lo más impresionante de su labor filosófica y es este mismo impulso el que nos hace preguntar por cuál sería la definitiva conclusión de su pensamiento. La concepción de la filosofía primera de X. Zubiri como una filosofía de la realidad trascendental (I. Ellacuría), como una noología (D. Gracia, A. Pintor-Ramos) o como una filosofía de la alteridad radical de todos los actos humanos (A. González) es todavía una cuestión disputada.

\section{Realismo metafísico y realismo zubiriano}

Si en X. Zubiri parecía que se disolvía el "escándalo de la filosofía" , el que la realidad del mundo exterior haya que admitirse meramente en virtud de una creencia, releyendo a X. Zubiri desde estas mismas sesiones, el "escándalo" parece que reaparece con toda su crudezas. No se trata ciertamente ya del hiato idealista entre alteridad del pensar y realidad, sino del hiato entre alteridad sentiente ("reidad") y realidad allende la aprehensión. Pero quizás esta impre- 
sión de hallarnos en la filosofía zubiriana ya instalados en la realidad obedecía a la misma equivocidad del término "realidad", porque propiamente donde estamos siempre es en la "reidad" o formalidad de realidad de todos los actos humanos, incluidos los racionales, y la razón lo que hace es profundizar en esta "reidad".

Desde su obra última, $X$. Zubiri no puede pronunciarse sobre aquello que sobrepasa el análisis de los actos ni puede descartar rotundamente diferentes explicaciones metafísicas posibles: que haya cosas independientes de toda aprehensión y que coincidan con lo que aprehendemos, que no las haya, o que la realidad en sí sea algo inalcanzable. El "reísmo" o realismo trascendental zubiriano no se preocupa tanto de buscar una prueba de que exista efectivamente o "realmente", independientemente de nuestros actos, la alteridad o "de suyo" deslindado en los actos como de mostrar que exista o no exista no tenemos más remedio en los actos racionales que considerar las cosas en su alteridad real. En los actos racionales no se actualiza propiamente lo que las cosas son con independencia de cualquier acto, sino lo que las cosas pudieran ser más allá de lo actualizado en aprehensión. No se actualiza la realidad misma, sino solamente aquello que pensamos que es la realidad con independencia de todo acto. La formalidad de realidad resulta intrascendible y sólo se da realidad, se defina como se defina, inscrita en la alteridad radical de todos los actos humanos. "El realismo trascendental" zubiriano no puede establecer dos zonas de cosas: la de la alteridad de los actos humanos y la alteridad independientemente de todo acto humano. Esto, en todo caso, son hipótesis metafísicas tan provisionales y necesarias como cualquier hipótesis científica.

Siempre pudiera suceder que la alteridad real de los actos racionales se resolviera en ser una alteridad con pretensión de realidad. Pero aunque en nuestros actos racionales llegáramos a actualizar como fondo de lo real o objeto de la razón la nada, la alteridad real permanece incancelable: "En rigor, el término pudiera ser un término vacío, esto es, el «hacia» sería un hacia nada. Sin embargo, siempre será «realmente» una nada; está por tanto en la realidad como un hueco suyo, por así decirlo" (IRA 139). Ciertamente, en mis actos se presenta la alteridad como realidad "o de suyo", pero nada garantiza que más allá de esta alteridad constitutiva de mis actos haya eso que comúnmente se llama realidad. ¿No sería, entonces, la filosofía primera de Zubiri idealista y se acabaría hundiendo en el precipicio del solipsismo? ¿Cómo funda Zubiri una trascendencia allende la aprehensión?

Pues mostrando cómo en la aprehensión accedemos al ámbito trascendental de la realidad. El orden transcendental no es, para X. Zubiri, el orden de la verdad ni del objeto ni del ente ni del ser, sino el orden de la realidad sentida en aprehensión. Es un orden o una dimensión que no es independiente de cada cosa. X. Zubiri no se cansa de advertir que no está hablando de la realidad como 
un piélago. La realidad no es una especie de infinitud hipostasiada que se contrae en los límites de cada cosa real. Tal realidad no es sino una abstracción. La realidad es sólo y siempre la realidad de cada cosa real. Su estar como "de suyo" en la aprehensión. Este "estar" de cada cosa es justo el que abre el ámbito trascendental, un ámbito previo a todo aquende y allende, a todo dentro y fuera. Las cosas quedan como realidad, pero podría no haber nada más allá de su estar como "de suyo" en la aprehensión y de su constitutivo remitir allende lo inmediatamente aprehendido en la aprehensión. Sin embargo, aunque en esta remisión no llegáramos a nada, aunque por diversos actos de razón llegáramos a la conclusión de que la realidad profunda de las cosas y de mí mismo es, en último término, evanescente y de que los contenidos de cada cosa no son más que alucinación, sueño o engaño, nada de esto negaría que estamos constitutivamente instalados en una realidad primordial, antes que en la subjetividad o en la nada misma. Ambas son mucho más problemáticas y construidas que aquella.

Así, realidad en X. Zubiri es aquello que la inteligencia sentiente aprehende en su primario, más humilde y elemental acto. Esta afimación no tiene nada que ver con el realismo metafísico. Es importante repetirlo una y otra vez, pues el uso técnico que hace X. Zubiri del término realidad como "reidad" o simple ser "de suyo" y su uso común en la historia de la filosofia, da pie a continuos malentendidos. El realismo metafísico es propiamente pre-fenomenológico y precrítico, mientras que la conceptuación de X. Zubiri es post-fenomenológica y postheideggeriana. Que la cosa aprehendida como real tenga existencia o sea una ilusión, que tengan tal o cual esencia, todo eso, con la importancia que pueda tener, no pasa de ser un problema ulterior. Lo primario consiste en la aprehensión de las cosas como reales. Lo que la inteligencia diga o deje de decir sobre lo real será también siempre ulterior: será logos y razón. Sin embargo, al ser la alteridad el carácter más elemental del sentir humano y, por ende, de la intelección, no podrá anularse jamás. La alteridad de la formalidad de realidad podrá recubrirse con las modalizaciones ulteriores de la intelección, pero no podrá zanjarse. El logos y la razón podrán intentar todo lo que quieran reducir fenomenológicamente, integrar conceptualmente en un sistema total o superar dialécticamente esta alteridad. Todo será en vano, por más que se intente violentar el sentir humano, éste no dejará de reivindicar sus fueros.

\section{Zubiri y la fenomenología}

$X$. Zubiri insiste, en la segunda sesión, en desmarcarse de toda fenomenología justamente porque pone entre paréntesis el momento de realidad a beneficio de un saber absoluto ${ }^{\circ}$. No hay problema en entender como fenomenológica la filosofía primera de X. Zubiri, si ésta se concibe en un sentido lato como descripción de hechos dados por sí mismos, prohibiéndose el paso a cualquier teoría (sea científica o metafísica) que vaya más allá de lo dado. Tampoco lo hay si se entiende la fenomenología como una peculiar manera de hacer filosofía, esto es, 
como una filosofía primera o fundamental a la búsqueda permanente de su propio objeto.

Sin embargo, se traicionaría la filosofía zubiriana si al tildarlo de fenomenólogo se entendiera por ello la tesis de que el plano original y decisivo de su análisis filosófico es el ámbito consciente. Ni sus conceptos básicos ni su ámbito de análisis son los propios de una filosofía del sentido. Cabría aún preguntarse si una filosofía que realiza los ideales de la fenomenología, dejando de lado la primacía del sentido y la conciencia, no podría legítimamente llamarse fenomenología, pero tajantemente asegurará Antonio Pintor-Ramos que un análisis que se mueva más allá del orden del sentido ya no puede con propiedad considerarse un análisis fenomenológico' ${ }^{7}$. Podrá discutirse si la filosofía primera de X. Zubiri es un análisis trascendental de la realidad, una noología o una filosofía de la acción humana, pero en ningún caso se trata de una fenomenología en su sentido estricto. Es más, después de haber dedicado sendos estudios a la cuestión, A. PintorRamos considera que si bien la filosofia primera de X. Zubiri es incomprensible sin la fenomenología, "etiquetarla" como fenomenológica introduce muchas más confusiones que claridad. Si efectivamente entendemos como A. Pintor-Ramos que la línea divisoria entre lo que puede considerarse o no fenomenología reside en el sentido, se comprende por qué X. Zubiri considera a Scheler y Heidegger como fenomenólogos y a él mismo no ${ }^{8}$.

$X$. Zubiri recalca en que no aprehendemos inmediatamente cosas-sentido, sino cosas-realidad. "Se nos dice (Husserl, Heidegger, etc.) que lo que formalmente aprehendemos en la percepción son, por ejemplo, paredes, mesas, puertas, etc. Ahora bien, esto es radicalmente falso. En una aprehensión impresiva yo no intelijo jamás una mesa. Lo que aprehendo es una constelación de notas que en mi vida funciona como mesa. Lo que aprehendo no es mesa sino una constelación de tal dimensión, forma, peso, color, etc., que tiene en mi vida función o sentido de mesa. Al aprehender lo que llamamos mesa, lo aprehendido como «de suyo» o "en propio» no es, pues, la mesa como mesa. La mesa no es «de suyo» mesa. La mesa es mesa tan sólo en cuanto la cosa real así llamada forma parte de la vida humana. Las cosas como momentos o partes de mi vida son lo que yo he llamado «cosa-sentido». Pero nada es «de suyo» cosa-sentido. La cosa real aprehendida como algo «de suyo" no es una "cosa-sentido", sino lo que he llamado "cosa-realidad»" (IRE 59). De ahí que la cuestión del sentido ocupe un lugar marginal en la obra de X. Zubiri.

\section{Inmediatez y sentir kinestésico}

Otros temas no menos importantes que aparecen en estas sesiones es la extrema importancia que cobra el sentir kinestésico y lo que debe entenderse por inmediatez. Por el sentir kinestésico, la realidad queda aprehendida direccionalmente, lanzándonos "allende lo aprehendido" (ese allende puede ser hacia dentro 
o hacia fuera) y manteniéndonos consecuentemente en una especie de tensión dinámica. Así, contra el idealismo el punto de partida no es la "representación"y, sino una realidad fisica que está ya en la más elemental sensación. Este momento de "hacia" incoado en toda aprehensión nos lleva a los modos ulteriores de intelección (logos y razón). Todo lo más la "representación" sería un momento provisional de la intelección destinada a desaparecer en la direccionalidad física y constitutiva de la intelección. Del mismo modo, este momento del "hacia" le permite enfatizar a X. Zubiri, más allá de los planteamientos de la filosofía modema, que la reflexión no sólo no es el acto primario de la intelección, ni un acto inmediato, sino que tampoco es un acto ajeno al sentir o a la sensibilidad humana (IRE 108). La reflexividad está ya incoada en el sentir kinestésico.

Respecto a la inmediatez es importante resaltar que desde Descartes el idealismo ha entendido que hay unos actos, los actos de intelección, que son transparentes a sí mismos. La piedra angular del idealismo es, de algún modo, la supuesta inmediatez del pensamiento frente a la mediatez del mundo. La conciencia sería así una especie de evidencia primera. X. Zubiri pretende desbancar al idealismo no apelando a otra hipótesis metafísica, sino por la insuficiencia de su punto de partida. Si de inmediatez se quiere hablar, lo único inmediato para $X$. Zubiri es la formalidad de realidad. Pero la inmediatez de esta formalidad está muy lejos de la pretendida autotrasparencia y evidencia de la conciencia para sí misma. La reflexión en $X$. Zubiri no es un acto inmediato ni primario, sino que está fundado en lo único inmediato, a saber: el mero estar en la realidad. No se arranca de la autotransparencia de ningún acto, sino de la apertura de un acto a los demás por la constitutiva formalidad de realidad. Tan inmediato es el acto de aprehensión como lo actualizado en el mismo. El acto de actualización no tiene originariamente ningún privilegio sobre lo actualizado en el mismo. En el punto de partida de X. Zubiri no hay una transparencia absoluta, pero al haber, en el permanente fluir y la extraordinaria exuberancia de los actos, un ámbito de trascendentalidad, tampoco son éstos totalmente opacos, podemos desprender de ellos algunas esquirlas de inteligibilidad volviendo a través de actos reflexivos sobre los actos mismos (NHD 150).

\section{Ciencia y filosofía}

$X$. Zubiri explica a I. Ellacuría que no se puede pretender que la metafísica sea una especie de marco trascendental absoluto o menos variable que el de las construcciones científicas, que tendrían lugar en su seno. "Es la misma la relatividad en la metafísica que en la física"'". La filosofía es un volver con nuestros actos reflexivos sobre nuestros actos anteriores, colocando "en nueva perspectiva el mundo entero de nuestra vida, incluyendo los objetos y cuantos conocimientos científicos hayamos adquirido sobre ellos" (NHD 150). "La reflexión indica aquí solamente este acto de volver sobre nuestros actos y no un acto de 
meditación o de introspección como cuando se habla de conciencia refleja, por oposición a la conciencia directa" (NHD 150)". Es esta diferencia radical de perspectiva la que impide utilizar el método y los resultados de la ciencia como objeción al saber filosófico. Si la mayor riqueza que nos brindan los saberes científicos es la permanente profundización en el conocimiento de determinadas realidades, la mayor riqueza que quizás cabe esperar del conocimiento filosófico es la persistente remoción de prejuicios en función de una descripción más rigurosa de los actos humanos sobre los que vuelve la filosofía. La repercusión más decisiva del saber filosófico sobre los demás saberes probablemente sea la de posibilitar una continua depuración del aparato conceptual y metafísico de los mismos.

La necesidad de liberar a la ciencia de prejuicios metafísicos es precisamente lo que destaca X. Zubiri en su ensayo de 1935: "La idea de naturaleza: la nueva física" (NHD 291 ss). No sería exagerado decir que la física que se constituye a partir de Plank, Schrödinger, Heisenberg, Didac ${ }^{12}$ puede constituir una buena introducción al núcleo de la filosofía zubiriana. Algunas de las nociones centrales de la metafisica zubiriana pueden ser muy bien aproximadas pedagógicamente desde los estudios de la física ${ }^{13}$, pero sobre todo lo que nos muestra la nueva física es la necesidad de pensar la realidad de las cosas sin la rémora de la metafísica griega que sigue empalagosamente constriñendo las creaciones científicas (NHD 333)" ${ }^{14}$. X. Zubiri dirá que "la nueva física pone en vibración, a un tiempo, el cuerpo entero de la filosofía" (NHD 295). No se trata de que la filosofía tenga que montarse sobre los resultados de la ciencia, sino de que las mismas nociones y convenciones filosóficas, al igual que las de la física, deben ser abandonadas cuando no expresan la realidad. La aportación decisiva del filosofar es precisamente la permanente remoción de los prejuicios metafísicos con que cargan las diferentes áreas del saber, incluyendo lo más difícil de todo que es barrer en la propia casa del saber filosófico. La fecundidad de este empeño nos la muestra, por ejemplo, Diego Gracia en ética y en biología; I. Ellacuría al tratar de pensar La historia fuera del cañamazo de las nociones modemas, y A. Gonź́lez en ecciología y teología ${ }^{15}$.

Ya se ve, entonces, lo equívoco que puede ser destacar que la filosofía de $\mathbf{X}$. Zubiri es importante en la medida en que se ciñe a los conocimientos científicos para la elaboración filosófica. Ciertamente en el transcurso de su vida, los saberes matemáticos, físicos, biológicos y neurobiológicos cobran una gran importancia, mucho más que los saberes sociales. Podrá, incluso, decirse que X. Zubiri trata do pensar el ámbito trascendental que abren las cosas desde las nociones de algunas hacias contemporáneas más que desde la poesía o la metáfora, como hiciera Hidegger, pero no se circunscribe ni mucho menos al conocimiento científico, id Este es necesariamente el más importante. Una novela, la poesía, una película - una danza pueden decimos más sobre la esencia de algunas cosas que las construcciones científicas (IRA 123-127): "El poeta poetiza porque las cosas le 
dan que pensar. Y esto que así piensa de ellas es su poesía. Que lo inteligido así sea una realidad teoréticamente conceptuada o sea realidad en ficción, o sea realidad poética, no cambia la esencia de la intelección como razón (IRA 42).

$X$. Zubiri cuestiona ya, desde su inicio, en el filosofar el prejuicio, decisivo en la modernidad, de que la filosofía ha de emular el saber científico: Descartes, Hegel, Kant y Husserl encarnarían en uno u otro sentido esta ambición de dotar a la filosofía de un método seguro y productivo como el de las demás ciencias (NHD 145-147). "La objeción contra la filosofía procede de una cierta concepción de la ciencia que, sin previa discusión, pretende aplicarse unívocamente a todo saber estricto y riguroso" (NHD 147). Un científico de lo primero que suele quejarse al acercarse a la filosofía es que "se pierde en los abismos de una discordia" (NHD 144) donde no cabe punto de encuentro. Pero para X. Zubiri, el escepticismo y la impresionante diversidad y discordia de las posiciones filosóficas no son más que el zaguán de la filosofía. La filosofía empieza justamente cuando vencemos estas "dos tendencias antagónicas que surgen espontáneamente en un espíritu principiante: la de perderse en el escepticismo, o la de decidirse a adherir polémicamente a una fórmula con preferencia a otra, tratando incluso de forjar una nueva. Dejemos estas actitudes para otros" (NHD 144). Y es que "no hay que esperar de la filosofía que nos cuente, por ejemplo de las fuerzas físicas, de los organismos o de los triángulos, nada que fuera inaccesible para la matemática, la física o la biología. Nos enriquece llevándonos a otro tipo de consideraciones" (NHD 149). Cuando nos decidimos a abandonar la antecámara de la filosofía y penetrar en su recinto, accedemos a un punto de encuentro universal, a una consideración trascendental de las cosas, no por trascendental y universal menos física, inmediata, abierta, histórica y provisional que las construcciones científicas.

\section{Etapas del pensamiento de X. Zubiri}

I. Ellacuría establece tres etapas en el pensamiento de X. Zubiri. La primera etapa tendría el sello del catedrático de Historia de la filosofía y se cerraría con el abandono de X. Zubiri de la universidad y con la publicación de Naturaleza, Historia, Dios, en 1944. Esta fase ya se diferenciaría por su preocupación por ir más allá del ser y del sentido en busca de la realidad, pero sin una tematización clara de sus estructuras trascendentales. Una segunda etapa de maduración empezaría en 1945 y concluiría en 1962 con la publicación de Sobre la esencia, y se caracterizaría por presentar como determinante del quehacer filosófico el análisis trascendental de la realidad misma. Y la tercera etapa o etapa madura arrancaría en 1962, y en ella la consideración trascendental de la realidad, frente a las consideraciones positivo-talitativas, ocuparía decisivamente el primer lugar ${ }^{16}$. El problema de esta división es que no está nada claro que antes de 1961 fueran las consideraciones positivo-talitativas las que ocuparan el primer lugar para $\mathrm{X}$. Zubiri. De hecho, en la mayor parte de los cursos inéditos de 1945 a 1962 creo 
que de lo que se trata es de poner en marcha, en la consideración de los problemas, la posición ganada en su filosofía primera. Por otro lado es probable que a partir de 1962 haya cambios y precisiones terminológicas más decisivas en la evolución del pensamiento zubiriano, que el que pueda suponer la publicación de Sobre la Esencia.

Si en un primer momento, en los cursos que empieza a brindar $\mathrm{X}$. Zubiri en 1945 -después de Naturaleza, Historia, Dios- se intenta proyectar luz desde el ámbito radical ganado en su filosofía primera hacia la antropología, la metafísica y la teología, a sus setenta años $X$. Zubiri cada vez evita más cualquier rodeo metafísico o consideración antropológica para mantenerse en el análisis de lo inmediatamente dado en aprehensión. I. Ellacuría tenía en mente un esquema lineal de la evolución del pensamiento de X. Zubiri. X. Zubiri habría pasado de una consideración positivo-talitativa de las cosas, que sería el propio de las ciencias, a una consideración o conceptuación de la talidad en cuanto tal ya propiamente metafísica y, de allí, a una consideración trascendental de la realidad. Sin embargo, creo que un esquema de maduración por el que X. Zubiri se va afirmando y haciendo fuerte en el punto de partida radical, logrado en su última etapa mediante "salidas" cada vez más acotadas, hasta concentrarse en su obra última en el mero análisis de este punto de partida, retrata más fielmente el quehacer filosófico de Zubiri. La realidad dada en impresión es, desde 1945, el punto de partida de Zubiri. De modo que puede decirse que, desde entonces, los diferentes cursos y publicaciones son no tanto un rodeo por la antropología, la metafísica o las diferentes ciencias, sino una puesta en marcha y una proyección en otras esferas de la radicalidad alcanzada en el punto de partida de su filosofía.

\section{La perspectiva de I. Ellacuría}

Creo que I. Ellacuría vislumbró con claridad toda la novedad y potencia crítica y creadora que almacenaba la última filosofía de X. Zubiri: "Quienes critican el pensamiento de $\mathrm{X}$. Zubiri sin conocerlo y sin capacidad de contraponerle uno propio, harían bien en sobrepasar tópicos sociologizantes para penetrar en lo que tiene de rigor, de novedad y de potencialidad crítica y creadora, incluso para temas sociológicos. X. Zubiri, como cualquier otro filósofo creativo, no está conforme con ninguna de las filosofías pasadas; tampoco con la suya. Pocos menos conservadores que él; pocos tan críticos como él. Pero su crítica se basa en que ve la realidad de otra forma y es capaz de pensarla, de conceptuarla. Es crítico porque es creativo y la crítica le lleva a la creación filosófica"17. Pero sea por intereses metafísico-históricos y teológicos, por no considerar relevante en la conflictividad social latinoamericana la última obra de X. Zubiri, por dar por bueno el análisis último de $\mathbf{X}$. Zubiri y aprovecharlo para elaborar una antropología y una metafísica, o simplemente por la falta de tiempo para replantear Sobre la esencia a la luz de Inteligencia Sentiente, creo que nunca llegó a instalarse en el ámbito radical en el que acaba viviendo la filosofía de X. Zubiri y 
desde el que proyecta y revisa las diferentes ramas del saber filosófico. De hecho, en el último artículo que publicó I. Ellacuría sobre X. Zubiri, no está claro que I. Ellacuría no incurra en este equívoco mortal del que nos previene $\mathbf{X}$. Zubiri en estas sesiones: "Cuando yo dije que aquí hay un equívoco mortal, me refería a que se puede pensar que las "cosas" son cosas fuera de la percepción, y que luego no hacen sino actualizarse en la percepción. Claro esto no lo diría yo jamás. Es un equívoco mortal"'s.

Incluso, en el último texto que escribió I. Ellacuría sobre X. Zubiri parece estar presente este equívoco: "La formalidad de realidad nos abre así al ámbito de las cosas reales en tanto que reales. Si la realidad es una formalidad, las cosas reales no son una formalidad, sino que son simplemente cosas reales. La formalidad de realidad que viene últimamente de las cosas reales se presenta en ellas de un modo peculiar. Aunque provisionalmente puede definirse lo real, como lo físico (SE, 11-13), como «todo y sólo aquello que actúa sobre las demás cosas o sobre sí mismo en virtud, fornalmente, de las notas que posee» (SE, 104), la realidad misma de las cosas reales ha de medirse - la realidad tiene grados- por el «de suyo». Así la mesa no es "de suyo» mesa, sino un conjunto de notas físicas, que en cuanto tales sí son reales, pero que hacen de la mesa meramente una cosasentido, algo que desde sus propiedades reales tiene un sentido para la vida del hombre. Así no son igualmente reales las notas constitucionales y las adventicias, las notas constitutivas o esenciales que las meramente constitucionales. $Y$ tampoco tiene el mismo grado de realidad la piedra que el animal, el animal que el hombre, porque el «de suyo» que les corresponde es distinto hasta llegar en el hombre a convertirse su «de suyo» físico en un físico «suyo», lo cual le permite ser reduplicativamente suyo. Por ello, la formalidad de realidad se da también en las cosas reales, que son "de suyo» lo que son y lo son a su modo"14.

En esa línea prolongará siempre I. Ellacuría a X. Zubiri: "Como la realidad no nos es presente sino en las cosas reales, y como las cosas reales que nos son presentes como reales a una inteligencia sentiente son las cosas de este mundo, no hay otro comienzo para una filosofía primera que el de realizar una metafísica intramundana"21). El análisis de la realidad intramundana, en cuanto realidad, es el interés de I. Ellacuría. Una filosofía de la naturaleza y una filosofía de la historia, una filosofía del hombre y una filosofía de la sociedad son, claro está, importantísimas y decisivas para dar algunas respuestas a los problemas de nuestro tiempo, pero la partida de todas ellas parece que se juega en la filosofía primera. Mientras la metafísica tiene la misma relatividad que una teoría científica ${ }^{21}$, la filosofía primera goza de una menor apertura. En este ámbito de radicalidad habita por estos lares A. González, y su apasionante libro es una muestra de los tesoros escondidos y lo fructífera que puede ser para los diferentes saberes la prosecución de la misma ${ }^{22}$.

Una cuestión en la que la posición de I. Ellacuría parece más certera que la de X. Zubiri es en lo que el sentido común entiende por "realidad"23. No es un 
problema nada banal. Si haciendo caso a lo que nos dice el mismo X. Zubiri en este texto al precisar la noción de "inmediatez"2A a las palabras hay que conservarles un poco el sentido que tienen so pena de escribir un lenguaje que no es accesible para nadie, tendremos que repensar la propiedad de la palabra "realidad" para designar al hecho de que las cosas queden "de suyo" en la aprehensión.

Hay, sin duda, muchas más cosas relevantes en estas sesiones. Quizás lo más importante es que descubrimos en ellas la auténtica pasión, y vocación filosófica de X. Zubiri y de I. Ellacuría. Su actitud filosófica es de tal calibre, que lejos de descansar en cualquier ortodoxia o fosilización de nociones parecen siempre dispuestos a desmentirse a sí mismos, en el permanente y siempre abierto proceso de la radicalización filosófica. Hoy, ante el testimonio de estas discusiones, podemos y debemos reivindicar punto por punto el talante que preconizaba I. Ellacuría al presentar, en 1977, la actualidad de X. Zubiri: "La filosofía puede y debe ser criticada por sus insuficiencias sociales, por su carácter de ideología; pero, ante todo, debe ser criticada por sus insuficiencias filosóficas. Las insuficiencias sociales las deben estudiar, en primer lugar, los sociólogos; las filosóficas, los filósofos. Y uno se teme que sean pseudo-filósofos los que están haciendo la crítica pseudo-social del pensamiento de X. Zubiri. Esperemos que tras la etapa, a veces muy necesaria y fructuosa, de la sofística, venga la etapa de la construcción filosófica, que responda a la altura de nuestro tiempo. [...] Ahora que podemos, no dejemos que se grite el "que filosofen ellos""2s. La radical eficacia y poder liberador de la filosofía se da sobre todo cuando ésta es fiel a sí misma.

\section{Notas}

1. Cf. [2. 3. El realismo zubiriano frente al idealismo y al realismo ingenuo y crítico.]

2. A. Pintor-Ramos, Génesis y formación de la filosofía de Zubiri, op. cit., p. 103 y ss; D. Gracia, Voluntad de verdad, op. cit., p. 112.

3. J. Bañón, "Zubiri hoy: Tesis básicas sobre la realidad", Del sentido a la realidad, Trotta, 1995, p. 92.

4. I. Kant, Crítica de la razón pura, B XXXIX.

5. Cf. [2.5. ¿Anfibología del término realidad?]

6. Cf. [3.3. Diferencias con la fenomenología]

7. A. Pintor-Ramos, Realidad y verdad, Salamanca, 1994, p. 321 y ss.

8. X. Zubiri, "Sobre el problema de la filosofía", en la Revista de Occidente 115 (1933) 51-80 y 118 (1933) 83-117.

9. Cf. [2.7. El sesgo peculiar del realismo zubiriano]

10. Cf. [2.2. Relatividad de la formalidad de realidad y de la metafísica]

11. Esto que aparece como programa en Naturaleza, Historia. Dios se cumple definitivamente en el análisis de la inteligencia sentiente que realiza en su última obra y es, desde este último análisis, desde el cual mide Zubiri los conceptos que constituyen el sistema de la metafísica (PFMO 343). Es importante destacar que X. Zubiri considera que tan ingénita y espontánea es esta perspectiva filosófica como la de los demás saberes (NHD 150). 
12. X. Zubiri, "La idea de naturaleza: La nueva física", NHD 293 y ss. X. Zubiri considera que Einstein es la última expresión de una física aún imbuida por una concepción mecanicista de la naturaleza. Es lo que lleva a Einstein a rebelarse contra la nueva física: "Pensar que en cada instante está Dios jugando a los dados con todos los electrones del universo, esto, francamente, es demasiado ateísmo" (NHD 351). Diego Gracia replicará que el problema está en que el Dios al que se refiere Einstein es más el Dios de Espinosa que el de las religioncs, y que quienes piensan así, siempre han querido ver la naturaleza desde el punto de vista de la divinidad. Diego Gracia, "Problemas filosóficos en genética y en embriología", La mediación de la filosofia en la construcción de la bioética, Universidad Complutense, Madrid, 1994, p. 219.

13. Nociones decisivas en la filosofía zubiriana como las de "campo" en sustitución de "cosa" o "elemento", "función" en sustitución de "causa", "estructura" en sustitución de "substancia", "apertura" en sustitución de determinismo y desarrollo, "sustantividad" en sustitución de esencia, "sistema" en sustitución de conjunto, y "nota" en sustitución de "propiedad" son perfectamente inteligibles desde la física contemporánea.

14. Un ejemplo de este constreñimiento estaría en la biología mucho más pegada hasta principios de los setenta a una concepción aristotélica y tradicional de la naturaleza. Diego Gracia, op. cil., p. 217 y ss.

15. D. Gracia, Fundamentos de bioética, Madrid, 1989; I. Ellacuria, Filosofía de la realidad histórica, Madrid, 1991; A. González, Un solo Mundo, la relevancia de Zubiri para la teoría social, tesis doctoral, Comillas, Madrid, 1995; A. González, "Aproximación a la filosofía de la religión de Xavier Zubiri", Realidod, 63, mayojunio, San Salvador, pp. 293-306.

16. I. Ellacuría, "Introducción crítica a la antropología de X. Zubiri", Realitas II, Madrid, 1975 p. 82 y ss.

17. I. Ellacuría, "Actualidad de la filosofía zubiriana" 1977. Este párrafo no aparece en Ya, pero se encuentra en el borrador original del artículo y aparecerá en la edición de las obras completas de I. Ellacuría.

18. [2.3. El realismo zubiriano frente al idealismo $y$ al realismo ingenuo y crítico]

19. I. Ellacuría, "La superación del reduccionismo idealista en X. Zubiri", ECA, 477, 1988, pp. 633-650.

20. I. Ellacuria, La idea de filosofla en homenaje a X. Zubiri, Ed. Moneda y Crédito, Vol. 1, Madrid, 1970, pp. 459-523.

21. Cf. [2.2. Relatividad de la formalidad de realidad y de la metafísica]

22. A. González, Estructuras de la praxis, Ensayo de uno filosofla primera, Trotta, 1997.

23. [3.10. Noción filosófica de realidad y sentido común]

24. Cf. [1.4. Inmediatez y sentir cenestésico]

25. I. Ellacuría, “Actualidad de la filosofía zubiriana", Ya, Madrid, 1977. 